\title{
Spontaneous regression of a big BCC. Is there a role for aloe verra?
}

\author{
Stefanaki $C^{1 *}$, Plaka $M^{1}$, Skylakaki $M^{2}$, Theologi $B^{3}$, Rigopoulos $D^{1}$ and Stratigos $A^{1}$ \\ ${ }^{1} 1$ st Department of Dermatology- Venereology, National and Kapodestrian University of Athens School of Medicine, Andreas Sygros Hospital, Athens, Greece \\ ${ }^{2}$ Radiology Department, Evangelismos General Hospital, Athens, Greece \\ ${ }^{3}$ Pathology Department, National and Kapodestrian University of Athens School of Medicine, Andreas Sygros Hospital, Athens, Greece
}

\begin{abstract}
A 95-year-old man presented with a $10 \mathrm{~cm}$ mass on the forehead with a central ulceration and a rolled edge, hard and fixed with underlying tissues present for 9 months. Histopathological examination revealed a typical basal cell carcinoma with metatypic features. Computed tomography of the head excluded infiltration of the bone and underlying tissues. We also detected two pearly nodular lesions on the left cheek and a similar on the nose suggestive of bccs. The patient was advised to start vismodegib but he refused and decided to start a herbal medicine based on aloe verra (one leaf per day) mixed with the juice of one pomegranate and one orange daily. After 3 months on the herbal juice the big lesion and all other tumors on the patient's face regressed totally. Interestingly, he reported that regression of the big lesion halted when he stopped the medication for 10 days.
\end{abstract}

A 95-year-old man presented with a mass on the forehead of 9 months duration. The mass was mainly asymptomatic but was growing rapidly over a period of 6 months. He had cryotherapy for the lesion 6 months ago, which resulted in a central non healing ulcer, without halting its progression. The patient's mental and physical health was excellent, and he was not on any regular medication. He reported surgical excision of two basal cell carcinomas (BCC) one from the left ear and one from the side of the nose few years ago.

On examination we detected a huge tumorous mass on the forehead (Figure 1) measuring $10 \mathrm{~cm}$, with a central ulceration, an indurated base, and a big, rolled edge at the periphery. The mass was fixed with the underlying tissues and was hard and non-tender on palpation. Dermoscopy of the rolled edge revealed linear arborizing vessels but no maple leaf-like structures. We also detected another three suspicious lesions on the patient's face, two on the left cheek and one on the right side of the nose. The lesions on the cheek were nodular with a pearly appearance and overlying telangiectasias, whereas on the nose had a pearly border and a depressed center.

A punch biopsy was taken from the border of the mass and histopathological examination revealed a typical basal cell carcinoma with metatypic features (Figure 2) and the diagnosis of basosquamous BCC was established. The patient underwent a computed tomography of the head, where infiltration the bone, of the underlying tissues and of cervical or preauricular lymph nodes were excluded. The three smaller tumors on the patient's face were diagnosed as BCCs based only on clinical features.

Given that surgery was not feasible for the huge tumor and impractical for all the BCCs on the patient's face and radiotherapy was excluded given the metatypic features of the big tumor, the patient was advised to start vismodegib. He refused and decided to start a herbal medicine based on aloe verra (one whole leaf per day) mixed with the juice of one pomegranate and one orange daily. After 3 months on the herbal juice the big lesion and all other tumors on the patient's face regressed totally (Figure 3). Interestingly, he reported that regression of the big lesion halted when he stopped the medication for 10 days. He underwent another computed tomography of the scalp were no progression of the big tumor on the forehead was detected. The patient refused to have another biopsy in order to detect any residual tumor.

Spontaneous regression of BCCs has been previously described and immune response is thought to play a primarily role through $\mathrm{CD} 4+\mathrm{T}$ lymphocytes that release cytokines such as INF- $\gamma[1,2]$. Similarly, incompletely excised BCCs have been shown to regress and no residual tumor has been reported upon re-excision in $24-42 \%$ of cases. However, spontaneous regression of a big tumor such the one in our patient has not been previously reported. Although regression of our patient BCCs has not been confirmed histologically regression is beyond doubt based on clinical data. It is unlikely that cryotherapy triggered an immune response and subsequent regression given that the tumor had been growing for months after cryotherapy. Interestingly tumor regression was arrested when our patient stopped taking his juice with aloe $[3,4]$.

Aloe verra contains pharmacologically active ingredients that demonstrate diverse biological activities including immunomodulating and anticancer effects [5]. Particularly one active ingredient aloe emodin has been shown to possess anti-proliferative effects and to induce cellular apoptosis, thus demonstrating anti-cancer activity against a variety of tumors including neuroectodermal, nasopharyngeal cancer, lung squamous cell carcinoma, hepatocellular carcinoma, prostate and

*Correspondence to: Christina Stefanaki, 13 Pontou street, Ilisia 11527, Athens, Greece, Tel: +30210-7487822, Fax: +30210-7487822, Email: cstefana@otenet.gr

Key words: basal cell carcinoma, regression, metatypic, aloe verra

Received: August 10, 2020; Accepted: August 20, 2020; Published: August 26, 2020 


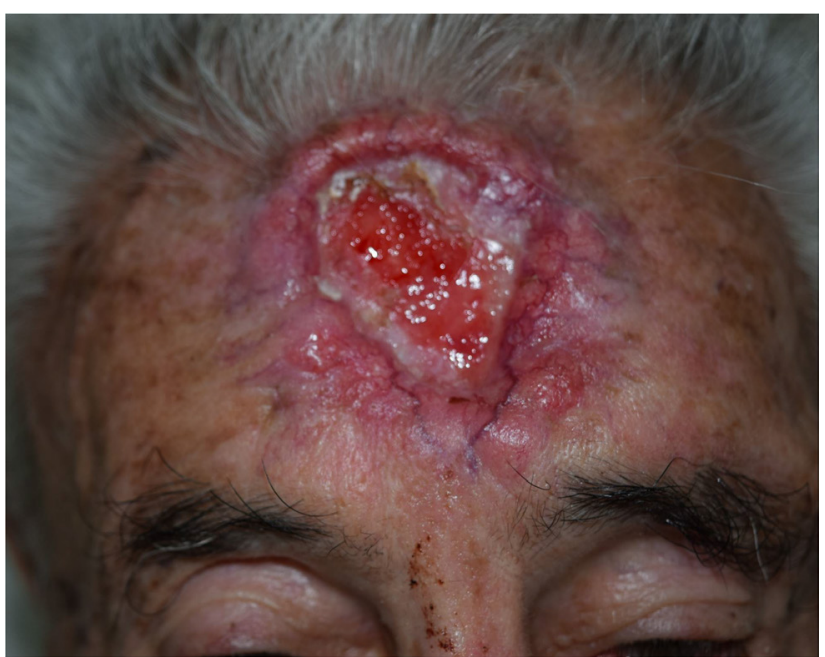

Figure 1. A huge metatypic bcc on the patient's forehead

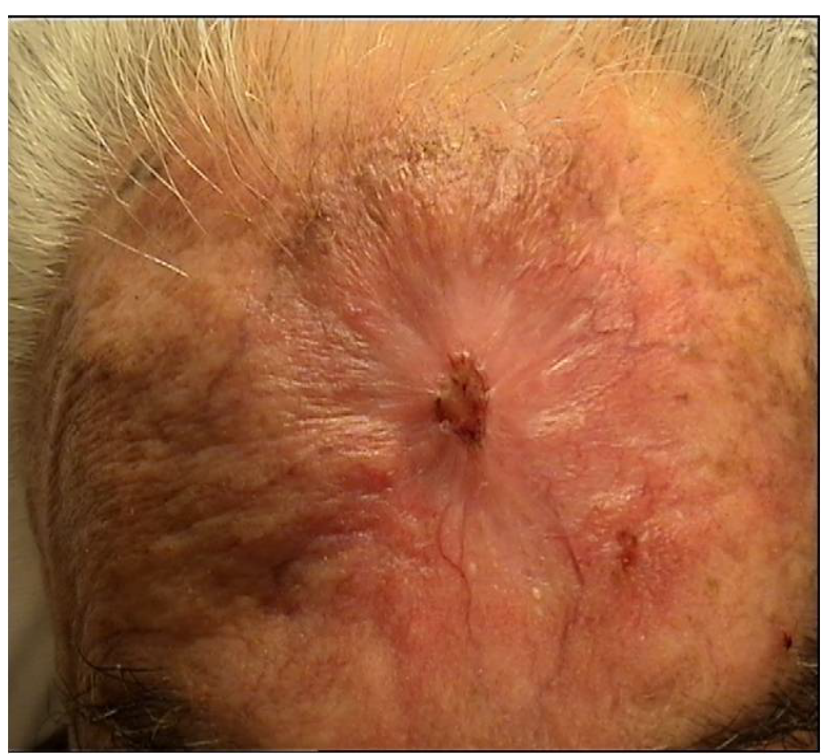

Figure 2. Clinical regression of the mass on the forehead after aloe treatment

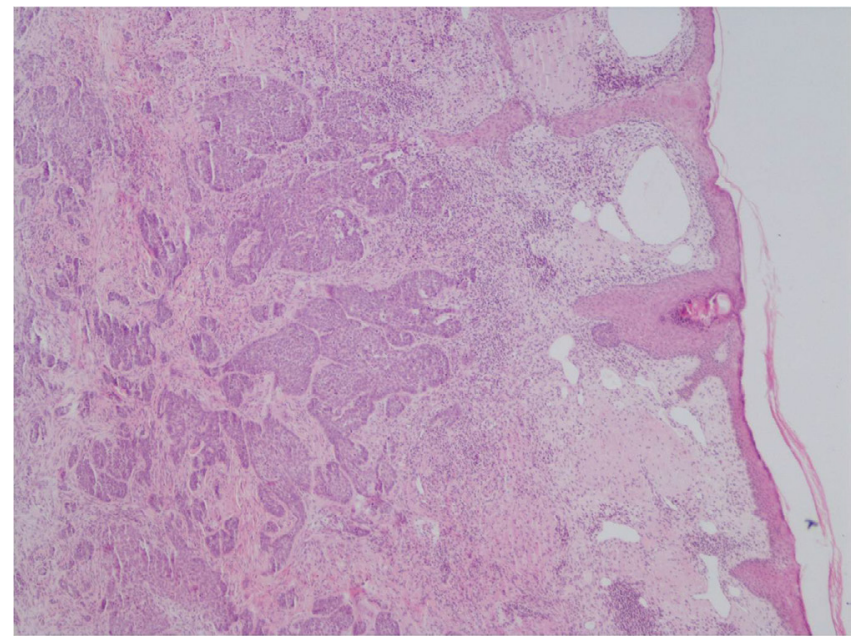

Figure 3. Histological picture of the tumor on the forehead showing nests of neoplastic cells in the mid and deep dermis with peripheral palisading without any considerable detachment of the dermis, compatible with metatypical bcc gastric cancer [6]. Combined with conventional chemotherapy has improved survival in patients with a variety of metastatic tumors and has been shown to reduce the incidence of chemotherapy associated toxicity [7]. Studies have shown that aloe exerts its anti-cancer activity through apoptotic cell death by oxidative stress and sustained JNK activation, whereas others have demonstrated cell death through $S$ phase arrest [6]. Aloe verra has never been investigated for the treatment of BCC and our patient's case may prompt further studies on the role of aloe in inducing BCC regression.

\section{Acknowledgement}

No funding sources for this work.

\section{Conflicts of interest}

No

\section{References}

1. Wong DA, Bishop GA, Lowes MA, Cooke B, Barnetson RS, et al. (2000) Cytokine profiles in spontaneously regressing basal cell carcinomas. Br J Dermatol 143: 91-98. [Crossref]

2. Hunt MJ, Halliday GM, Weedon D, Cooke BE, Barnetson RS (1994) Regression in basal cell carcinoma: an immunohistochemical analysis. Br J Dermatol 130: 1-8. [Crossref]

3. Stewart CM, Garlick J, Mcmullin J (2015) Surgical Excision of non-melanoma skin cancer in an elderly veteran's affairs population. Plast Reconstr Surg Glob Open 2: e277. [Crossref]

4. Rieger KE, Linos E, Egbert BM, Swetter SM (2010) Recurrence rates associated with incompletely excised low-risk nonmelanoma skin cancer. J Cutan Pathol 37: 59-67. [Crossref]

5. Guo X, Mei N (2016) Aloe vera: A review of toxicity and adverse clinical effects Journal of Environmental Science and Health 34: 77-96. [Crossref]

6. Zhang LQ, Lv RW, Qu XD, Chen XJ, Lu HS (2017) Aloesin suppresses cell growth and metastasis in ovarian cancer SKOV3 cells through the inhibition of the MAPK signaling pathway. Anal Cell Pathol 2017: 8158254. [Crossref]

7. Cathcart P, Stebbing J (2016) Aloe vera, a natural cancer soother? Lancet Oncol 17: 421. [Crossref]

Copyright: (C2020 Stefanaki C. This is an open-access article distributed under the terms of the Creative Commons Attribution License, which permits unrestricted use, distribution, and reproduction in any medium, provided the original author and source are credited. 